\title{
A CHARACTERIZATION THEOREM FOR BOUNDED STARSHAPED SETS IN THE PLANE
}

\author{
MARILYN BREEN
}

\begin{abstract}
Let $S$ be a nonempty bounded set in $R^{2}$. Then $S$ is starshaped if and only if every 3 or fewer boundary points of $S$ are clearly visible via $S$ from a common point of $S$. The number 3 is best possible.
\end{abstract}

1. Introduction. We will need the following definitions from [1 and 2]. Let $S$ be a subset of $R^{d}$. A point $s$ in $S$ is said to be a point of local convexity of $S$ if and only if there is some neighborhood $N$ of $s$ such that $N \cap S$ is convex. If $S$ fails to be locally convex at point $q$ in $S$, then $q$ is called a point of local nonconvexity (lnc point) of $S$. For points $x$ and $y$ in $S$, we say $x$ sees $y$ via $S$ ( $x$ is visible from $y$ via $S$ ) if and only if the corresponding segment $[x, y]$ lies in $S$. Similarly, for $x$ in $\operatorname{cl} S$ and $y$ in $S, x$ is clearly visible from $y$ via $S$ if and only if there is some neighborhood $N$ of $x$ such that $y$ sees each point of $N \cap S$ via $S$. Finally, set $S$ is called starshaped if and only if there is some point $p$ in $S$ such that $p$ sees each point of $S$ via $S$, and the set of all such points $p$ is called the (convex) kernel of $S$, denoted $\operatorname{ker} S$.

A well-known theorem of Krasnosel'skii [6] states that if $S$ is a nonempty compact set in $R^{d}$, then $S$ is starshaped if and only if every $d+1$ points of $S$ are visible from a common point of $S$. Moreover, a stronger result may be obtained by replacing points of $S$ with boundary points of $S$. Unfortunately, these theorems fail for noncompact sets (examples in [3] reveal that no such theorem is possible without some modifications), and the problem of obtaining a Krasnosel'skii-type theorem to characterize noncompact starshaped sets remains an intriguing one. It is this type of characterization theorem which will be considered here. An important tool will be the concept of clearly visible, a notion which has appeared in papers by Stavrakas [7] and Falconer [4]. Recently (in [1 and 2]) analogues of the Krasnosel'skii theorem were obtained by replacing the concept of visible with that of clearly visible and by replacing points of $S$ with points of local nonconvexity of $S$. Here this kind of replacement is used again to obtain the following result: Let $S$ be a nonempty bounded set in $R^{2}$. Then $S$ is starshaped if and only if every 3 or fewer boundary points of $S$ are clearly visible via $S$ from a common point of $S$. The number 3 is best possible.

As in [1 and 2], the following familiar terminology will be used: Conv $S, \operatorname{cl} S, \operatorname{int} S$, rel int $S$, bdry $S$ and ker $S$ will denote the convex hull, closure, interior, relative interior, boundary, and kernel, respectively, for set $S$. Lnc $S$ will be the set of points of local nonconvexity of $S$, and if $S$ is convex, $\operatorname{dim} S$ will be the dimension of $S$. For distinct points $x$ and $y, R(x, y)$ will represent the ray emanating from $x$ through

Received by the editors October 10, 1984.

1980 Mathematics Subject Classification. Primary 52A30, 52A35; Secondary 52A10.

Key words and phrases. Starshaped sets, convex kernel, Krasnosel'skii-type theorems. 
$y$, and $L(x, y)$ will be the line determined by $x$ and $y$. The reader is referred to Valentine [8] for a discussion of these concepts.

2. The results. We will be concerned with the proof of the following theorem.

THEOREM 1. Let $S$ be a nonempty bounded set in $R^{2}$. Then $S$ is starshaped if and only if every 3 or fewer boundary points of $S$ are clearly visible via $S$ from a common point of $S$. The number 3 is best possible.

ProOF. The necessity of the condition is obvious, so we need to establish only its sufficiency. Assume that set $S$ satisfies the condition stated above. Then certainly $S$ is a bounded connected set in $R^{2}$, and every 3 or fewer lnc points of $S$ are clearly visible via $S$ from a common point. Hence we may repeat the construction in $[\mathbf{2}$, Theorem A] to select a point $p$ in $S$ having the following properties: Point $p$ is in (ker cl $S) \cap S$, the corresponding set $A \equiv\{s: s \in S$ and $[p, s] \nsubseteq S\}$ is nowhere dense in $S$, and $A \subseteq$ bdry $S$.

The proof of the theorem will be accomplished by considering cases determined by the arrangement of points in $(\operatorname{int} \operatorname{cl} S) \sim S$. For future reference, we remark that by [2, Lemma 3], every point in (int cl $S) \sim S$ lies in a nondegenerate segment in (int $\operatorname{cl} S) \sim S$. There are three possibilities to consider.

Case 1. Assume that $(\operatorname{int} \operatorname{cl} S) \sim S=\emptyset$. We examine subcases determined by the dimension of $\operatorname{ker} \operatorname{cl} S \neq \emptyset$.

Case 1a. In case $\operatorname{dim}(\operatorname{ker} \operatorname{cl} S)=0$, then point $p$ defined above is the unique member of $\operatorname{ker} \operatorname{cl} S$. We will show that $p \in \operatorname{ker} S$ as well. Let $x$ be an arbitrary point of $S$ to prove that $[p, x] \subseteq S$. If $x \in \operatorname{int} S$, then since $A \subseteq$ bdry $S$, the result is immediate. If $x \in \operatorname{bdry} S$, assume on the contrary that there is some point in $(p, x) \sim S$ to reach a contradiction. Choose points $z, z_{0}$ on $[p, x]$ such that all points between $p$ and $z$ (if any exist) are in $S$, all points between $z_{0}$ and $x$ (if any exist) are in $S$, and the corresponding lengths of $[p, z]$ and of $\left[z_{0}, x\right]$ are maximal. Then $z, z_{0} \in$ bdry $S$, and using the facts that $p \in \operatorname{ker} \operatorname{cl} S$ and (int $\left.\operatorname{cl} S\right) \sim S=\emptyset$, it is easy to see that $[z, x] \subseteq$ bdry $S$.

By a similar argument, $z_{0}$ is clearly visible via $S$ only from points in one of the closed halfplanes determined by the line of $L \equiv L(p, x)$, say $\operatorname{cl} L_{1}$. Let $R_{1}$ be a ray emanating from point $p, R_{1} \subseteq L_{1} \cup\{p\}$. If every boundary point of $\operatorname{cl} S$ were clearly visible via cl $S$ from some point of $R_{1} \sim\{p\}$, then using the fact that bdry $S$ is compact, methods used in [2] would yield a nondegenerate segment at $p$ in $R_{1}$ such that every boundary point of $\operatorname{cl} S$ could see each point of this segment via $\operatorname{cl} S$. But then this segment would be in $\operatorname{ker} \operatorname{cl} S$, impossible since $\operatorname{ker} \operatorname{cl} S$ contains only point $p$. Thus, there exists a boundary point $b_{1}$ of $\operatorname{cl} S$ not clearly visible via $\mathrm{cl} S$ from any point of $R_{1} \sim\{p\}$. Furthermore, we assert that $b_{1}$ and $z_{0}$ together are clearly visible via $S$ only from points in one of the closed convex regions bounded by $R_{1}$ and $L$ :

Suppose on the contrary that $b_{1}$ and $z_{0}$ are clearly visible via $S$ from points $s$ and $t$ in opposite open halfplanes determined by the line of $R_{1}$. Then $s, t \in \operatorname{cl} L_{1} \sim R_{1}$. Without loss of generality, assume that $t$ and $z_{0}$ are on the same side of $R_{1}, s$ and $z_{0}$ on opposite sides. Certainly $s \notin L$ since $z_{0}$ is clearly visible via $S$ from $s$. Consider in turn each of the following possible locations for point $b_{1}: b_{1} \in L_{1}$, $b_{1} \in R\left(p, z_{0}\right), b_{1} \in R\left(z_{0}, p\right), b_{1} \in L_{2}$. In each case, select a convex neighborhood $N$ of $b_{1}$ so that both $s$ and $t$ see via $\operatorname{cl} S$ every point of $N \cap \operatorname{cl} S$. Let $\left(s, z_{0}\right) \cap R_{1}=\left\{p^{\prime}\right\}$ 
and $(s, t) \cap R_{1}=\left\{p^{\prime \prime}\right\}$. Since $s \notin L$, neither $p^{\prime}$ nor $p^{\prime \prime}$ is $p$. Let $p_{0}$ be the member of $\left\{p^{\prime}, p^{\prime \prime}\right\}$ closer to $p$. Using the facts that $\left[s, z_{0}\right] \subseteq \operatorname{cl} S$ and $p \in \operatorname{ker} \operatorname{cl} S$, it follows that points of $\left(p, p_{0}\right)$ see via $\mathrm{cl} S$ each point of $N \cap \operatorname{cl} S$. However, $b_{1}$ is not clearly visible via cl $S$ from any point of $R_{1} \sim\{p\}$, so this is impossible. We conclude that $b_{1}$ cannot be in any of these locations. Our supposition is false, and $z_{0}$ and $b_{1}$ together are clearly visible via $S$ only from points in one closed convex region bounded by $R_{1}$ and $L$. Let $T_{1}$ denote this region and let $R_{0}=L \cap$ bdry $T_{1}$.

Continuing, let ray $R_{2}$ emanate from $p$ and bisect the angle determined by $R_{0}$ and $R_{1}$. Using the argument above, select a boundary point $b_{2}$ of $\operatorname{cl} S$ such that $b_{2}$ is not clearly visible via $\mathrm{cl} S$ from any point of $R_{2} \sim\{p\}$. Then $b_{2}$ and $z_{0}$ are clearly visible via $S$ only from points in one of the closed convex regions, say $T_{2}$, bounded by $R_{2}$ and $L$. Furthermore, points $b_{1}, b_{2}$ and $z_{0}$ are clearly visible via $S$ only from points in $T_{1} \cap T_{2}$ which is bounded by $R_{2}$ and one of $R_{0}, R_{1}$. By an obvious induction, we obtain a sequence of boundary points $\left\{b_{n}\right\}$ and corresponding sequences of rays $\left\{R_{n}\right\}$ and regions $\left\{T_{n}\right\}$ such that the following are true: For $n \geq 2$, point $b_{n}$ is not clearly visible via $\operatorname{cl} S$ from any point of $R_{n} \sim\{p\}$, ray $R_{n}$ bisects the angle determined by $T_{1} \cap \cdots \cap T_{n-1}$, and region $T_{1} \cap \cdots \cap T_{n}$ is bounded by $R_{n}$ and one of $R_{0}, R_{1}, \ldots, R_{n-1}$. Observe that if $T_{1} \cap \cdots \cap T_{n}$ is bounded by $R_{n}$ and $R_{j}$ for $j \geq 1$, then $b_{n}, z_{0}, b_{j}$ are clearly visible via $S$ only from points in $T_{1} \cap \cdots \cap T_{n}$. Similarly, if $T_{1} \cap \cdots \cap T_{n}$ is bounded by $R_{n}$ and $R_{0}$, then $b_{n}$ and $z_{0}$ are clearly visible via $S$ only from points in $T_{1} \cap \cdots \cap T_{n}$.

The sequence of rays $\left\{R_{n}\right\}$ converges to some ray $R$. Let $b$ be a limit point for $\left\{b_{n}\right\}$. Clearly, $b \in$ bdry $S$ since $\left\{b_{n}\right\} \subseteq$ bdry $S$. Now if $R=R_{j}$ for some $j \geq 1$, then it is easy to see that points $b, b_{j}, z_{0}$ can be clearly visible via $S$ only from points on $R_{j}$. But $b_{j}$ is not clearly visible via $\operatorname{cl} S$ from any point of $R_{j} \sim\{p\}$ and hence $b_{j}$ is not clearly visible via $S$ from any point of $R_{j} \sim\{p\}$. Since $z_{0}$ is not clearly visible via $S$ from $p$, points $b, b_{j}, z_{0}$ cannot be clearly visible via $S$ from any common point. We have a contradiction, and $R \neq R_{j}$ for any $j \geq 1$.

Similarly, if $R=R_{0}$, then $b$ and $z_{0}$ are clearly visible via $S$ only from points on $R_{0} \sim\{p\}$. Clearly such points lie on $R\left(p, z_{0}\right) \sim\left[p, z_{0}\right)$. Thus by our hypothesis for set $S$, every boundary point of $S$ is clearly visible from a point of $R\left(p, z_{0}\right) \sim\left[p, z_{0}\right)$. By standard arguments, we obtain a nondegenerate segment at $p$ in $\operatorname{ker} \operatorname{cl} S$. This violates our hypothesis for Case $1 \mathrm{a}$, and we conclude that $R \neq R_{0}$.

Since $R \neq R_{k}$ for any $k \geq 0$, there exist subsequences $\left\{R_{j}^{\prime}\right\},\left\{R_{j}^{\prime \prime}\right\}$ of $R_{k}$ which converge to $R$ and which lie in opposite closed half-planes $\mathrm{cl} H_{2}, \mathrm{cl} H_{1}$, respectively, determined by the line $H$ of $R$. Let $\left\{b_{j}^{\prime}\right\}$ and $\left\{b_{j}^{\prime \prime}\right\}$ denote the corresponding sets of boundary points of $S$. Since we may pass to convergent subsequences of $\left\{b_{j}^{\prime}\right\}$ and $\left\{b_{j}^{\prime \prime}\right\}$, for convenience of notation, assume that $\left\{b_{j}^{\prime}\right\}$ converges to point $b^{\prime}$ and that $\left\{b_{j}^{\prime \prime}\right\}$ converges to $b^{\prime \prime}$. Furthermore, assume that $H_{1}$ and $H_{2}$ are labeled so that $z_{0} \in H_{1}$. Then $b^{\prime}$ and $z_{0}$ are clearly visible via $S$ only from points in $\operatorname{cl} H_{1} \cap \operatorname{cl} L_{1}$, $b^{\prime \prime}$ and $z_{0}$ are clearly visible via $S$ only from points in $\operatorname{cl} H_{2} \cap L_{1}$, and of course $b^{\prime}, b^{\prime \prime}, z_{0}$ are clearly visible via $S$ only from points in $R \sim\{p\}$.

We will prove that every boundary point of $S$ is clearly visible via $S$ from a point of $R \sim\{p\}$ : Let $y$ be an arbitrary boundary point of $S$. Points $y, b^{\prime \prime}, z_{0}$ are clearly visible via $S$ from some $v$, and by the remarks above, $v \in \operatorname{cl} H_{2} \cap L_{1}$. Similarly, points $y, b^{\prime}, z_{0}$ are clearly visible via $S$ from some $w$, and $w \in \operatorname{cl} H_{1} \cap \operatorname{cl} L_{1} \sim\left[p, z_{0}\right)$. If $v$ or $w$ is on $H$, then certainly $y$ is clearly visible from a point of $R \sim\{p\}$, the 
desired result. If $v \in H_{2}$ and $w \in H_{1}$, a geometric argument shows that $y$ is clearly visible via $S$ from some point of $R \sim\{p\}$, and again we have our result.

Thus every boundary point of $S$ is clearly visible via $S$ from some point of $R \sim$ $\{p\}$. Using the compactness of bdry $S$, standard arguments yield a nondegenerate segment in ker $\operatorname{cl} S$, again contradicting the fact that $\operatorname{dim} \operatorname{ker} \operatorname{cl} S=0$. Our original assumption must be false, $[p, x] \subseteq S$, and we conclude that $p \in \operatorname{ker} S$. In fact, it is easy to see that $\{p\}=\operatorname{ker} S$. This finishes the argument in Case 1a.

Case 1b. Suppose that $\operatorname{dim} \operatorname{ker} \operatorname{cl} S=1$. Let $G$ be the line determined by ker cl $S$. If for one open halfplane $G_{1}$ determined by $G$, every boundary point of $S$ is clearly visible via $S$ from a point of $G_{1}$, then standard arguments similar to those in [2] yield a 2-dimensional subset of $\operatorname{ker} \operatorname{cl} S$, impossible. Hence there exist boundary points $b$ and $c$ of $S$ such that $b$ is not clearly visible via $S$ from any point in $G_{1}$ and $c$ is not clearly visible via $S$ from any point in $G_{2}$.

We assert that $G \cap S$ is connected: Suppose not. Then $G \cap S$ has at least two components. For $u, v$ distinct endpoints of different components of $G \cap S, u$ and $v$ are in bdry $S$ and are clearly visible via $S$ only from points in $G_{1} \cup G_{2}$. Since $u, v, b$ are clearly visible from some common point of $S, G_{2} \cap S \neq \emptyset$. A similar argument for $u, v, c$ shows that $G_{1} \cap S \neq \emptyset$. Using this information, it is easy to prove that every relatively interior point of $\operatorname{ker} \operatorname{cl} S$ is in $\operatorname{int} \operatorname{cl} S$ and hence in $S$ (since (int $\operatorname{cl} S) \sim S=\emptyset$ ). We conclude that $\operatorname{rel} \operatorname{int}(\operatorname{ker} \mathrm{cl} S$ ) lies in one component $K$ of $G \cap S$. Let $y$ be an endpoint of $K$ and let $x$ be an endpoint of another component of $G \cap S$, with $x \notin \operatorname{cl} K$. Since (int $\operatorname{cl} S$ ) $\sim S=\emptyset$ and $x \notin \operatorname{cl} K$, it is easy to show that for at least one of the open halfplanes $G_{1}$ and $G_{2}$, say for $G_{1}, x$ fails to be clearly visible via $S$ from any point of $G_{1}$. Then $x$ and $c$ are clearly visible via $S$ only from points on line $G$, and the boundary points $x, y, c$ are clearly visible via $S$ only from points on $G$. However, this implies that $(x, k] \subseteq S$ for some $k \in K$, so $(x, k] \subseteq K$, contradicting the fact that $x \notin \mathrm{cl} K$. Our supposition must be false, and $G \cap S$ is indeed connected.

Using this result, we are ready to prove that $S$ is starshaped. There are two possibilities to consider. In case rel int $(\operatorname{ker} \operatorname{cl} S) \cap S$ contains some point $q$, we show that $q \in \operatorname{ker} S$ : Select $s \in S$. If $s \in S \sim G$, then since (int $\operatorname{cl} S) \sim S=\emptyset$, it is easy to see that $[q, s] \subseteq S$. Likewise, if $s$ is in the connected set $G \cap S$, then $[q, s] \subseteq S$, so $q \in \operatorname{ker} S \neq \emptyset$.

In case $($ rel int $(\operatorname{ker} \operatorname{cl} S)) \cap S=\emptyset$, let $p$ be the point of $(\operatorname{ker} \operatorname{cl} S) \cap S$ described in our opening paragraph. Certainly $p$ must be an endpoint of ker $\operatorname{cl} S$. We show that $p \in \operatorname{ker} S:$ Again let $s \in S$. If $s \in G \cap S$, then $[p, s] \subseteq S$, so assume that $s \in S \sim G$. Ray $R(p, s) \sim[p, s)$ meets bdry $S$ at some first point $s^{\prime}$, and we note that if $s \neq s^{\prime}$, then $\left[s, s^{\prime}\right) \subseteq S$. Using our hypothesis for set $S$, boundary points $s^{\prime}, b, c$ are clearly visible via $S$ from some common point $t$, and by our choice of $b$ and $c, t$ must belong to $G \cap S$, which is connected and disjoint from relint $(\operatorname{ker} \operatorname{cl} S)$. If $t=p$, then $\left[p, s^{\prime}\right) \subseteq S$ and so $[p, s] \subseteq S$. If $t \neq p$, then $[t, p] \subseteq G \cap S \subseteq \sim \operatorname{relint}(\operatorname{ker} \operatorname{cl} S)$, and $t<p<r$ for $r \in \operatorname{rel} \operatorname{int}(\operatorname{ker} \operatorname{cl} S)$. Thus $(p, s) \subseteq \operatorname{int} \operatorname{conv}\left\{t, s^{\prime}, \operatorname{ker} \operatorname{cl} S\right\} \subseteq \operatorname{int} \operatorname{cl} S \subseteq S$, and $[p, s] \subseteq S$. Hence $p \in \operatorname{ker} S \neq \emptyset$, and we conclude that $S$ is starshaped, finishing the argument in Case 1b.

Case 1c. In case $\operatorname{dim} \operatorname{ker} \operatorname{cl} S=2$, select any point $q$ in $\operatorname{int}(\operatorname{ker} \operatorname{cl} S) \subseteq S$. For $s \in S$, it is easy to see that $[q, s) \subseteq \operatorname{int} \operatorname{cl} S$. Hence $[q, s] \subseteq S, q \in \operatorname{ker} S$, and $S$ is starshaped. 
This completes the proof in case $(\operatorname{int} \operatorname{cl} S) \sim S=\emptyset$. When $(\operatorname{int} \operatorname{cl} S) \sim S \neq$ $\emptyset$, each of its points lies in a nondegenerate segment in (int $\operatorname{cl} S) \sim S$, and the remaining cases will be determined by the arrangement of these segments.

Case 2. Assume that (int $\operatorname{cl} S) \sim S$ contains two or more noncollinear segments. Let $p$ be the point of $(\operatorname{ker} \operatorname{cl} S) \cap S$ described in our opening paragraph, with $A=$ $\{s: s$ in $S$ and $[p, s] \nsubseteq S\}$. By an easy adaptation of [2, Lemma 4], every boundary point of $S$ is clearly visible from $p$ via $S$, and $p$ is unique. Then since $A \subseteq$ bdry $S$, $A$ must be empty and $\{p\}=\operatorname{ker} S$.

Case 3. Assume that $(\operatorname{int} \operatorname{cl} S) \sim S \neq \emptyset$ has all its segments on line $J$, and let $T$ be a segment in (int $c l S) \sim S$ having maximal length. As usual, let $p$ and $A$ be the particular point and set defined in our opening paragraph. By $[2$, Lemmas 5 and 6], for one endpoint $t$ of $T, t$ is clearly visible via $S$ only from points in some interval $V$ at $t$, with $V \subseteq S$. Moreover, by the selection of point $p$ in [2, Theorem 2], $p \in V$. For future reference, notice that $t \in \operatorname{bdry} S$.

The following observation will be useful. For $u \in J \cap S$ and $v \in V \subseteq S$, we assert that $[u, v] \subseteq S$ : If $u=p$, the result is immediate. If $u \neq p$, the ray $R(p, u) \sim[p, u)$ meets bdry $S$ at some first point $u^{\prime}$, and if $u \neq u^{\prime}$, then $\left[u, u^{\prime}\right) \subseteq S$. Using our hypothesis for set $S, u^{\prime}$ and $t$ are clearly visible via $S$ from some point of $V$, so $\left[p, u^{\prime}\right) \subseteq S,[p, u] \subseteq S$, and $[v, u] \subseteq S$. Our assertion is established.

Now if for every $z$ in bdry $S \cap S,[p, z] \subseteq S$, then since $A \subseteq$ bdry $S, A$ must be empty, and $p \in \operatorname{ker} S$, finishing the argument in Case 3. Hence we assume that for some point $w_{0}$ in bdry $S \cap S,\left[p, w_{0}\right] \nsubseteq S$. By the observation above, $w_{0} \notin J$. Furthermore, since all segments in (int $\operatorname{cl} S) \sim S$ are on $J$ and $\left[p, w_{0}\right] \nsubseteq S$, it is not hard to show that $w_{0}$ must be clearly visible via $S$ only from points in one closed halfplane $\operatorname{cl} M_{1}$ determined by line $L\left(p, w_{0}\right) \equiv M$. Then boundary points $w_{0}$ and $t$ are clearly visible via $S$ only from points in $\left(\operatorname{cl} M_{1}\right) \cap V \sim\{p\}=M_{1} \cap V \neq \emptyset$. Again by our hypothesis for set $S$, every boundary point of $S$ is clearly visible via $S$ from some point of $M_{1} \cap V$, and using standard arguments, we obtain a nondegenerate interval $I$ at $p$ in $(\operatorname{ker} \operatorname{cl} S) \cap M_{1} \cap V \subseteq S$.

Let $q$ be a point in $\operatorname{rel} i n t I$. We will show that $q \in \operatorname{ker} S$. Choose $s \in S$. If $s \in S \sim J$, since (int $\operatorname{cl} S) \sim S \subseteq J$, it is easy to show that $[q, s] \subseteq S$. If $s \in J \cap S$, then by our previous observation, $[q, s] \subseteq S$. Hence $q \in \operatorname{ker} S \neq \emptyset$ and $S$ is starshaped, completing the argument in Case 3 and finishing the proof of the theorem.

It is not hard to construct examples which show that the number 3 in Theorem $\mathbf{1}$ is best possible. In particular, [1, Example 1] illustrates this.

Finally, it is interesting to note that Theorem 1 fails completely without the boundedness condition on set $S$, even when $S$ is closed. Consider the following useful example by Hare and Kenelly (which appears in [ $\mathbf{5}$ and $\mathbf{3}$, Example 5]).

EXAMPLE 1. Let $T_{n}=\{(x, y): n-1 \leq y \leq n, n \leq x+y\}$, and let $S=\bigcup T_{n}$. Then every finite subset of $S$ is clearly visible via $S$ from a common point, yet $S$ is not starshaped.

\section{REFERENCES}

1. Marilyn Breen, A Krasnosel skii-type theorem for points of local nonconvexity, Proc. Amer. Math. Soc. 85 (1982), 261-266.

2. ___ Points of local nonconvexity and sets which are almost starshaped, Geom. Dedicata 13 (1982), 201-213. 
3. __ The dimension of the kernel of a planar set, Pacific J. Math. 82 (1979), 15-21.

4. K. J. Falconer, The dimension of the kernel of a compact starshaped set, Bull. London Math. Soc. 9 (1977), 313-316.

5. W. R. Hare, Jr. and J. W. Kenelly, Intersections of maximal starshaped sets, Proc. Amer. Math. Soc. 19 (1968), 1299-1302.

6. M. A. Krasnosel'skii, Sur un critère pour qu'un domain soit étoilé, Mat. Sb. (61) 19 (1946), 309-310.

7. Nick M. Stavrakas, The dimension of the convex kernel and points of local nonconvexity, Proc. Amer. Math. Soc. 34 (1972), 222-224.

8. F. A. Valentine, Convex sets, McGraw-Hill, New York, 1964.

DEPARTMENT OF MATHEMATICS, UNIVERSiTy OF OKLAHOMA, NORMAN, OKLAHOMA, 73019 